\title{
Role of Charges of the Surface-grafted Polymer Chains for Aqueous Lubrication at a Nonpolar Interface
}

\author{
Troels Røn, Jan Busk Madsen, Nikolaos Nikorgeorgos and Seunghwan Lee \\ Dept. of Mechanical Engineering, Technical University of Denmark, DK-2800 Kgs. Lyngby, Denmark \\ ( Received July 20, 2014 ; Revised August 19, 2014 ; Accepted August 22, 2014 )
}

\begin{abstract}
Charged polymer chains, i.e., polyelectrolytes, are known to show superior aqueous lubricating properties compared to those of neutral polymer chains, especially in brush conformation. This is primarily because of the incorporation of a large amount of counterions within the polymer layers and the consequently increased osmotic pressure. However, this effect is active only when the polymer chains remain immobilized even under tribostress, which is not realistic for high-contact pressure tribological applications, especially when they are irreversibly immobilized on tribopair surfaces. In contrast, with free polymers, which can be included as surfaceactive additives in the base lubricant (water), long-term lubricating performance based on "self-healing" properties is readily expected. In order to assess whether the superior aqueous lubricating properties of polyelectrolyte chains are valid for free polymers too, this study reviews recent studies on the tribological properties of many charged biopolymer and synthetic copolymers at a nonpolar, hydrophobic interface. In contrast to the irreversibly immobilized polyelectrolyte chains, free polyelectrolyte chains show inferior aqueous lubricating properties compared to their neutral counterparts owing to charge accumulation and the consequently impeded surface adsorption on the nonpolar surface. Nevertheless, bovine submaxillary mucin (BSM), a representative biopolymer, shows a sufficiently effective surface adsorption and aqueous lubricating capabilities even at neutral $\mathrm{pH}$ without losing the polyanionic characteristics.
\end{abstract}

Keywords - polymer brush, charge, aqueous lubrication, polyelectrolyte

\section{Nomenclature}

$\mu:$ Coefficient of friction

\section{Introduction}

Recently, interests to utilize water as lubricant have been increasing for various reasons, such as growing emphasis on environmental preservation and sustainability, health and biomedical engineering, and biomimetic approaches in a broad range of science and engineering disciplines [1-5]. Despite several outstanding merits of water as lubricant, such as being abundant, economical, environment-friendly, and excellent coolant, it reveals a few critical drawbacks too; for

Corresponding author : seele@mek.dtu.dk Tel: 45-4523-2193, Fax: 45-4593-2193 example, water has much narrower range of operation temperature than oil-based lubricants and shows corrosion problem for many metallic materials. However, a most critical problem of water as lubricant is, in fact, related to handling of external pressure. Unlike oils, water has very low pressure coefficient of viscosity [6], and thus water cannot effectively increase its viscosity under elevated pressure under tribological contacts. Eventually, elastohydrodynamic lubrication (EHL) mechanism is generally excluded in aqueous lubrication. Several attempts have been made to overcome this drawback, and one of the most successful potentials was shown from polymer chains grafted at the rubbing interfaces [1,2]. Opposing surfaces with adsorbed polymers, or even better with brush-like polymer chains, reveal repulsive interaction due to excluded volume of polymers and osmotic pressure $[8,9]$. This effect was first discovered from a system of polymer 
chains in organic solvent $[7,10]$. As the effect is interinterplay between polymer brush and solvent, rather than either of both alone, it can be readily applicable to water and water-soluble polymer chains.

Among many parameters determining the efficacy of lubrication by polymer chains in aqueous condition, "charges" along the polymer chains, i.e. those from polyelectrolytes, have been recognized as a viable means to improve load-bearing capacity, primarily due to increased osmotic pressure within the brush by incorporating a large amount of counterions [11]. Superior lubricity of polyelectrolytes to neutral ones is confirmed from many studies [11-15]. However, a common requirement for this effect is that polymer chains should remain immobilized on surface throughout tribological contacts. In fact, a majority of studies for this topic have employed covalently bonding to graft polyelectrolyte chains onto the surface, and the applied load was very mild from surface force apparatus (SFA) [11, 12] or microtribometer [13, 14]. But, the stability of organic lubricant layers under tribological stress is practically limited, and whether polyelectrolyte chains can still reveal superior lubricity to neutral ones remained unexplored under the condition that they are employed as friction-reducing additives in base lubricant (water). In that case, additives are normally designed to adsorb onto tribopair surface spontaneously, and even reform the slippery layer when tribostress-induced wear of the film occurs in order to enable a long-term lubricating service. Thus, it is of the primary interest in this study to investigate if polyelectrolyte chains can reveal superior lubricity to neutral counterparts even in the form of "free" surface-adsorbing additives in aqueous environment.

\section{Research Method and Content}

\section{2-1. Bio- and synthetic polymers}

Bovine submaxillary mucin (BSM, type I-S) was purchased from Sigma Aldrich (Brøndby, Denmark) and used as received by dissolving in PBS buffer $(\mathrm{pH}$ 7.4). Synthetic polymers were either purchased from manufacturers or synthesized; silanized poly(ethylene glycol) (Si-PEG) and poly(l-lysine)-g-poly(ethylene glycol) (PLL-g-PEG) have been purchased from Nektar (Huntsville, AL) and SuSoS (Dübendorf, Switzerland), respectively. The molecular weight of PEG was ca. $5 \mathrm{kDa}$ for both cases. Amphiphilic diblock copolymers have been synthesized at the Danish Polymer Centre, Department of Chemical and Biochemical Engineering, DTU (Denmark). Diblock copolymers take the composition of A- $b$ - $\mathrm{B}$, where " $\mathrm{A}$ " represents hydrophilic block and " $B$ " represents hydrophobic block. "A" includes poly(ethylene glycol) (PEG), poly (acrylic acid) (PAA), and "B" includes polystyrene (PS), Poly(2-methoxyethylacrylate) (PMEA), poly(dimethylsiloxane) (PDMS), poly(n-butyl acrylate) (PnBA), and polycaprolactone (PCL). A number of diblock copolymers with the combination of A and B blocks were synthesized. Schematics of the diblock copolymers employed in this study are illustrated in Fig. 4 (section 3.3). The molecular weight of each block was aimed at $5 \mathrm{kDa}$, although exact values were somewhat different. Details on the synthetic steps and analysis of some of diblock copolymers are provided in a previous publication [16].

\section{2-2. Surface adsorption properties}

Surface adsorption of the polymers onto PDMS substrate was characterized by means of optical waveguide lightmode spectroscopy (OWLS). OWLS (Microvacuum, OWLS model 210, Biosense software ver. 2.6.10, Budapest, Hungary) is an optical, non-labeling technique to characterize the adsorption properties of macromolecules from liquid to interfacing solid surfaces $[17,18]$. OWLS is based on the in-coupling of incident linearly polarized (He-Ne, $633 \mathrm{~nm}$ ) with waveguiding layer $\left(\mathrm{Si}_{\mathrm{x}} \mathrm{Ti}_{\mathrm{y}} \mathrm{O}_{\mathrm{z}}\right)$ on glass substrate; at a specific incidence angle, total internal reflectance occurs and this incidence angle changes due to the changes in refractive index at the interface. Further details of the operational principles of OWLS are found in literature $[17,18]$. Adsorbed mass on the waveguide surface was estimated using Feijter's equation [19]. In order to emulate the surface of adsorption with the tribopair surface, the surface of waveguides were coated with a 
thin layer of PDMS by spin coating. Further details on this preparation are also available in a previous publication [16].

\section{2-3. Tribopairs and the surface treatments with polymer} chains

Stainless steel pin ( $3 \mathrm{~mm}$ in radius), glass substrate (borosilicate microscope glass), or PDMS silicone rubbers were employed as tribopair for macroscopic scale friction tests. Two component silicone kit (Syl$\operatorname{gard}{ }^{\circledR} 184$, Dow Corning) was employed in order to formulate pin (hemisphere with $3 \mathrm{~mm}$ in radius) and disk (30 $\mathrm{mm}$ in diameter and $5 \mathrm{~mm}$ in thickness) [16]. The tribopairs were modified with the polymer chains; for Si-PEG coating, glass substrate was immersed in toluene where Si-PEG is dissolved $(1 \mathrm{mg} / \mathrm{mL})$ overnight, and rinsed with ethanol [20]. The estimated thickness of Si-PEG film in dry condition as characterized by ellipsometry is ca. $2.2 \mathrm{~nm}$ [19]. For all the other polymers, when polymer solutions $(0.5$ or $1 \mathrm{mg} /$ $\mathrm{mL}$ ) were employed as lubricant, the polymers are expected to adsorb spontaneously onto the substrates based on, for example, electrostatic attraction for PLL$g$-PEG onto glass substrate, and hydrophobic interaction for the amphiphilic diblock copolymers onto PDMS surfaces. Rinsing of glass substrate immersed in PLL- $g$-PEG solution provided a coating of PLL- $g$ PEG film (ca. $1.4 \mathrm{~nm}$ in dry condition [19] on the substrate.

\section{2-4. Friction measurements}

Pin-on-disk tribometry (CSM Instruments, software ver. 4.4 M, Peseux, Switzerland) was employed to characterize the lubricating properties of biopolymers and synthetic polymers. The applied load was controlled with dead weight and was in the range of 1-5 $\mathrm{N}$. The sliding speed was varied by means of motor underneath the disk, and varied within $0.25 \mathrm{~mm} / \mathrm{s}$ to $100 \mathrm{~mm} / \mathrm{s}$. The lubricating properties of Si-PEG and PLL- $g$-PEG were assessed by the measurement of $\mu$ values from repeated revolutions on the sliding track (up to 50 revolutions, although only 20 revolutions are presented) in HEPES buffer or polymer solution. The lubricating capabilities of diblock copolymer were assessed by acquiring $\mu$ vs speed plots and comparing with those obtained from polymer-free buffer solution (PBS). For control tests, $\mathrm{pH}$ was lowered to 2-3 by addition of $\mathrm{HCl}$.

\section{Results and Consideration}

3-1. Reversibly vs irreversibly bound polymer chains under tribostress: a case of neutral copolymers

Approaches to graft polymer chain attachment onto surfaces can be classified in a few different ways. For example, "grafting-to" vs "grafting-from" approaches $[8,9]$ are the classification according to whether polymer chains pre-made and allowed to adsorb onto surface ("grafting-to") or polymerization starts from the surface ("grafting-from"). Alternatively, they can be classified as "chemisorption" vs "physisorption" depending on the bonding nature between the polymer chains and the underlying substrates. The important criterion in this study is to classify according to whether polymer chains are immobilized onto the surface reversibly or irreversibly. Irreversible immobilization is mostly achieved via covalent bonding, but can be obtained both from "grafting-from" [15] and "grafting-to" [12] approaches. A distinct advantage of irreversible immobilization method is the bonding strength and stability of polymer chain films, and thus it is expected to be more suitable for long-term and under harsher environment compared to reversible immobilization method.

For tribological applications, however, the concept of 'superior stability' by irreversible immobilization becomes somewhat obscure. An exemplary data for this discussion with neutral polymer chains is presented in Fig. 1.

In these plots, PEG chains are grafted onto the surface in two different ways, (a) covalently and "irreversibly" immobilized onto glass via silane bonding, or (b) PEG chains are co-polymerized onto polycationic poly(L-lysine) backbone as comb-like copolymer first ("PLL-g-PEG"), and then positively charged PLL backbone, consequently the copolymer PLL- $g$ - 

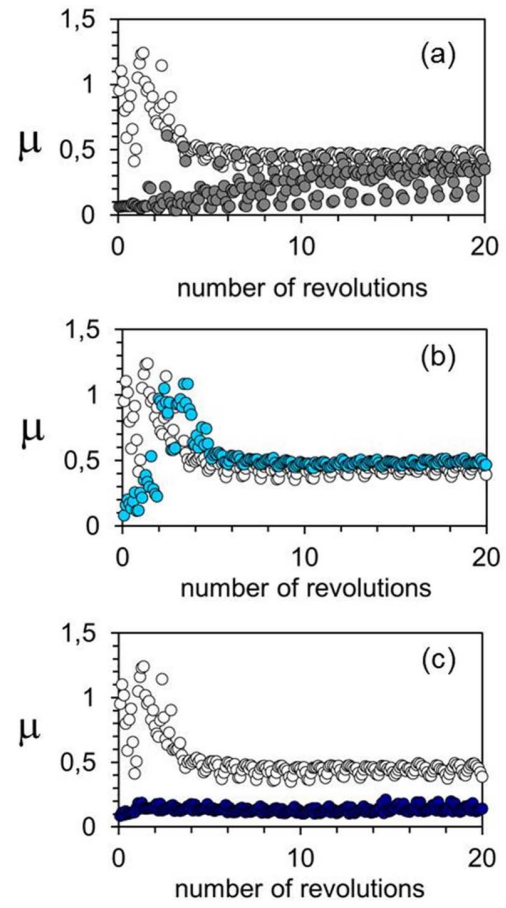

Fig. 1. $\mu$ vs the number of revolution plots from the sliding contact of a stainless steel pin (3 mm in radius) on the glass substrate ( $2 \mathrm{~N}, 5 \mathrm{~mm} / \mathrm{s})$ (a) Si-PEG coating on glass in HEPES, (b) PLL-g-PEG coating on glass in HEPES, (c) PLL-g-PEG solution $(0.25 \mathrm{mg} / \mathrm{mL})$ in HEPES. Blank symbols are for the data obtained from bare stainless steel vs glass contact in HEPES solution.

PEG overall, is attracted to negatively charged glass surface in neutral aqueous solution. Since covalent bonding, especially silane bonding, is much stronger than electrostatic attraction between $-\mathrm{NH}_{3}^{+}$(from lysine) and $\mathrm{SiO}^{-}$surface, higher stability is readily expected from Si-PEG than PLL- $g$-PEG. Under tribostress, for example, sliding against steel pin $(3 \mathrm{~mm}$ in radius, load $2 \mathrm{~N}$ ), even though both films showed gradual degradation of lubricating capabilities by increasing $\mathrm{m}$ values with accumulated tribostress (number of revolution), the rate of increase in $\mu$ is much slower for Si-PEG films than PLL-g-PEG; for PLL-g-PEG coating, the lubricating effect appears to cease only after 2 or 3 revolutions (Fig. 1(b)), whereas it took ca. 50 revolutions for Si-PEG film to indicate a complete removal (Fig. 1(a)) [20]. Nevertheless, the duration of the lubricating effect is extremely short even for Si-PEG films, and thus the efficacy of these films as practical means to lubricate hard materials is not promising. This is because irreversibly immobilized films are, regardless of its binding strength, removed from the surface by tribostress in an irreversible way too, and the recovery of the lubricating film is not achieved until the films are to be prepared from the beginning again. On the other hand, when PLL- $g$ PEG solution, as opposed to coating, is employed (Fig. 1(c)), the lubricating effect, $\mu$ being on the level of 0.1-0.2 compared to ca. 0.5 for bare tribopair, is persistent for much longer term; no increase in $\mu$ values was observed up to 50 revolutions (shown up to 20 revolutions in Fig. 1). This is likely to be from that the lubricating film by PLL- $g$-PEG is quickly reformed even if it is easily rubbed away as excess polymers present in bulk solution can quickly adsorb onto the area again. The exchange of the initially adsorbed PLL- $g$-PEG with excess PLL- $g$-PEG in solution was more vividly verified by labeling of the copolymer with different fluorescence tags [20]. It should be emphasized that the key parameter to ensure a persistent recovery of the lubricating films and long-term lubricating effect as in Fig. 1(c) is the fast surface adsorption kinetics rather than particular chemical bonding type or strength. Thus, the same argument can be applicable to the diblock copolymers to be addressed below.

\section{3-2. Biopolymers: Mucin}

As mentioned above, one of the momentums for recent growing interests in aqueous lubrication is increasing appreciation of remarkable performance of biological tribostystems, such as articular synovial joint systems, based on water as base lubricant. Many biopolymers are involved in the lubrication process of biological tissues and systems, such as lubricin, aggrecan, hyaluronan, and mucins. Their functions range from building up tissues of gliding interfaces, regulation of pressures in liquid, and finally to incorporation of water and lower interfacial friction forces as lubricant additive. Among them, mucin can be named as a 


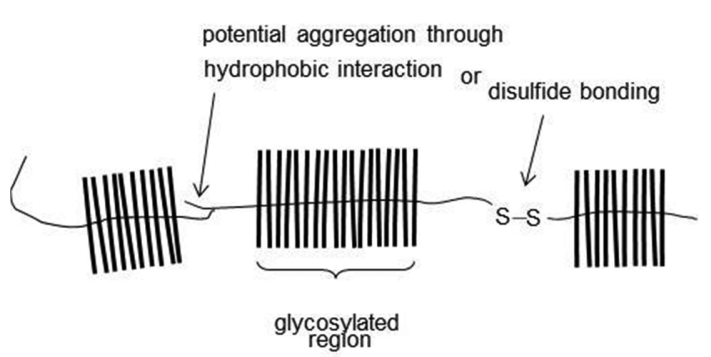

Fig. 2. A schematic illustration of mucins.

representative multifunctional biomacromolecule. Mucin is a high-molecular weight $(0.5-20 \mathrm{MD}[21,22])$, hierarchical polyanions, and is characterized for its heavy glycosylation at the central region [21,22]. A schematic of mucin structure is provided in Fig. 2.

Mucins primarily act as building block for secreted mucus, covering tissue surfaces interfacing with external environment, such as oculi, gastrointestinal, cervical, and respiratory organs, for protective and lubricating functions. Membrane-bound mucins are known to be involved in cell signaling and immunological defense as well [23].

The tribological properties of mucins are interesting at the gliding interfaces outside human or animal bodies too. This is due to that mucins display very distinct amphiphilic characteristic that they can readily adsorb onto hydrophobic substrates, as well as convert them hydrophilic for their capabilities to recruit a large amount of water at the interface [24]. A representative

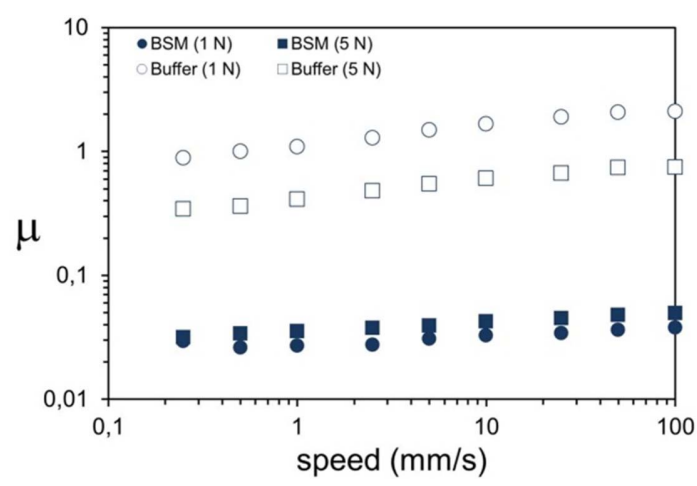

Fig. 3. $\mu$ vs speed plots obtained from BSM solution $(1 \mathrm{mg} / \mathrm{mL})$ at PDMS-PDMS sliding contact: 1 and 5 $\mathrm{N}$ load, radius of pin $6 \mathrm{~mm}$ in diameter. $\mu$ vs speed plots for BSM as characterized by pin-ondisk tribometry are presented in Fig. 3. In this plot, the tribo-interface was PMDS-PDMS, and the applied load was 1 or $5 \mathrm{~N}$.

As mentioned above, mucins are negatively charged at both glycans (sialic acids and sulfates) and unglycosylated C-and N-terminals regions. Thus, the adsorption of mucins onto surfaces generally follows those of polyanions. An important feature in this study is that adsorption of polyanions onto nonpolar, hydrophobic surface, such as PDMS, results in the accumulation charges on the surface, thus is impeded due to electrostatic charge repulsion between neighboring molecules on the substrate surfaces. This is a common feature of all charged species onto nonpolar surfaces $[25,26]$. Interestingly, BSM was found to be fairly effective in adsorption onto PDMS (ca. $>1 \mathrm{mg} / \mathrm{m}^{2}$ ) [27], and many other polymeric substrates, including PS, polyurethane, and poly(metharcrylic acid) (PMMA) [24]. For mucins, it is generally agreed that unglycosylated terminal region act as anchoring units, and thus interact with the hydrophobic substrates, whereas glycosylated central region interacts with water, stretching towards bulk water. Additionally, the lubrication of PDMS-PDMS sliding interface by BSM layer is quite effective, due to stable anchoring and effective incorporation of a large amount of water. As shown in Fig. 3 , the $\mu$ values of PDMS-PDMS sliding contact reduced from ca. 0.4-2.0 in PBS buffer (depending on the load) to ca. 0.02-0.04 in BSM aqueous solution $(1 \mathrm{mg} / \mathrm{mL}$ in PBS). As will be addressed below, the facile adsorption and lubrication of BSM at nonpolar interface are not readily reproduced by synthetic polymers. It is likely that the molecular weight, the ratio of anchoring/buoyant groups, and the charge characteristics of BSM etc. are optimized for the facile adsorption and lubrication at nonpolar interface.

The role of charges of BSM in the lubrication can be studied by lowering $\mathrm{pH}$, and consequently reducing the charges on the acidic moieties of BSM. A previous study [28] has shown that adsorbed mass of BSM increased at acidic $\mathrm{pH}$ compared to neutral $\mathrm{pH}$ (roughly by a factor of 2), and thus mechanically more 
stable films were generated at low $\mathrm{pH}$ condition. This is because BSM is also a polyanion and the reduction of charges along BSM at acidic $\mathrm{pH}$ facilitates the adsorption and aggregation of BSM on the nonpolar surface. This bears an important message that charges are rather impeding effective lubrication, as long as the tribopair surfaces are neutral and nonpolar. However, it is equally important to stress that the adsorption and aqueous lubricating properties of BSM at neutral $\mathrm{pH}$ condition is sufficiently good, as is shown in Fig. 3.

\section{3-3. Amphiphilic, diblock copolymers}

A very simplified emulation of mucin is diblock copolymers composed of hydrophobic and one hydrophilic block. The structure and composition of the diblock copolymers employed in this study are presented in Fig. 4.

As the diblock copolymers in Fig. 4 are amphiphilic, it can be expected that all of them may readily adsorb onto PDMS surface from aqueous solution. The contrasting buoyant blocks, PEG (neutral) vs PAA (polyanionic), were selected in order to investigate the influence of charges in these diblock copolymers on the adsorption and aqueous lubrication, for example, by directly comparing PEG- $b$-PS vs PAA- $b$-PS and PEG- $b$-PMEA vs PAA- $b$-PMEA. The representative results of $\mu$ vs speed plots for the diblock copolymers are presented in Fig. 5.

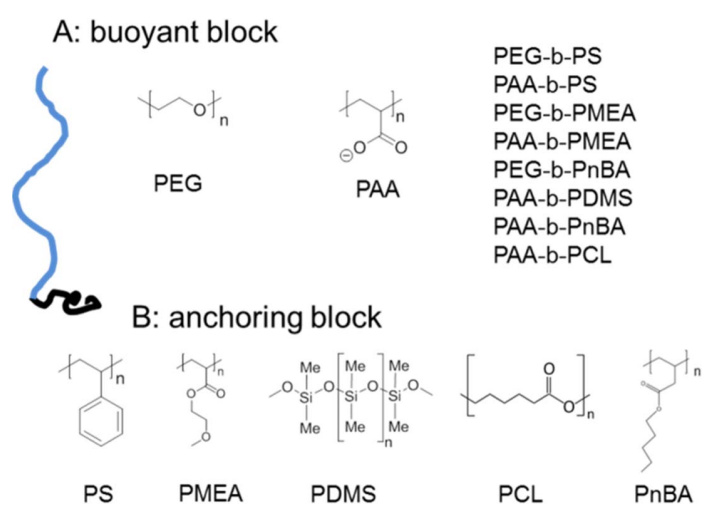

Fig. 4. Structure and composition of the amphiphilic diblock copolymers.

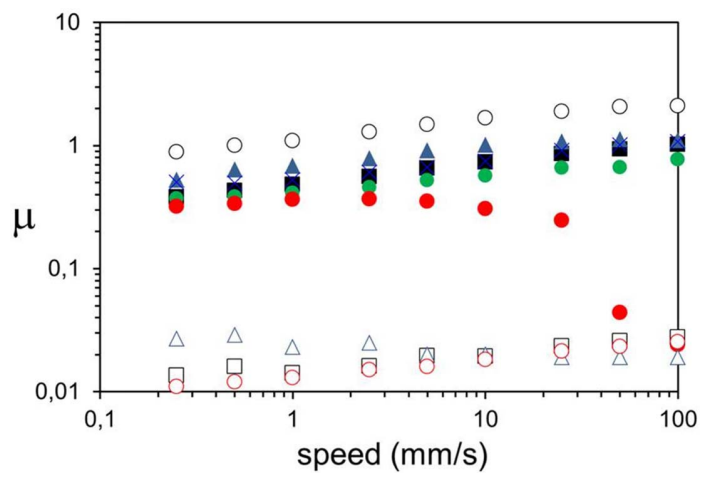

Fig. 5. $\mu$ vs speed plots for the diblock copolymers from PDMS-PDMS sliding contact, $5 \mathrm{~N}, 0.5 \mathrm{mg} / \mathrm{mL}$ polymer solution : blank circle for buffer solution, empty circle (red line) for PEG- $b$-PMEA, red-filled circle for PAA- $b$ PMEA, empty square (black line) for PEG-b-PS, blackfilled square for PAA- $b$-PS, empty triangle (blue line) for PEG- $b$-PnBA, blue-filled triangle for PAA- $b$-PnBA, cross for PDMS- $b$-PAA, and green-filled circle for PCL- $b$ PAA.

Interestingly, all the PEG-based, neutral diblock copolymers showed very effective lubricating properties (similarly with BSM in the previous section), whereas all the PAA-based, polyanionic counterparts showed not only inferior but also ineffective aqueous lubricating properties at PDMS-PDMS sliding contact. Inefficient lubricating capabilities of charged diblock copolymers can be readily explained by ineffective adsorption of polymers at nonpolar interface [16]. The relationship between the adsorption and lubricating properties of PS-based and PMEA-based copolymers were somewhat different though. For the former, adsorption itself was impaired and thus poor lubricity comes from the lack of lubricant films on the PDMS surface (adsorbed mass less than $0.1 \mathrm{mg} / \mathrm{cm}^{2}$ ). For the latter, while the adsorption was unignorable (on the level of ca. $0.8 \mathrm{mg} / \mathrm{m}^{2}$ [16]), they were very easily removed from the interface due to poor stability against tribostress. It is fairly clear that the major reason of poor adsorption and lubrication of PAA-based copolymers is the charged moieties, i.e. $\mathrm{COOH} / \mathrm{COO}-$ groups, as either lowering $\mathrm{pH}$ or increasing ionic strength improved surface adsorption as well as lubrication [16]. 
The results with the systematic variation of anchoring and buoyant block of the diblock copolymers support the results from BSM in that charged moieties along the polymer chains impede facile adsorption onto and aqueous lubrication of nonpolar, hydrophobic interfaces. A substantial difference though is that despite commonly improved lubricating properties of both types of polyanions (BSM and the PAA-based diblock copolymers) under "neutralized" condition (at low $\mathrm{pH}$ or high-salt condition), the PAA-based diblock copolymers have shown extremely poor lubricating performance at neutral $\mathrm{pH}$, whereas BSM is still providing a sufficiently good lubricity even at neutral $\mathrm{pH}$ condition.

3-4. Manipulation of polymer structure to improve the aqueous lubricating properties of charged polymers at neutral $\mathrm{pH}$ condition

Even if the lubricity of the polyanionic diblock copolymers can be improved by lowering $\mathrm{pH}$ or increasing ionic strength, neither of them provides a favorable environment for lubricating operation in general. Moreover, the motivation of employing charged copolymers, in fact, disappears in that condition. Instead of changing environment, optimizing the structure of copolymer could be a solution to this problem in order to operate the lubrication performance at neutral $\mathrm{pH}$ condition. As mentioned above, successful performance of BSM at neutral $\mathrm{pH}$ condition must be partly related to its optimum structure, in terms of molecular weight, charge ratio, and the ratio between hydrophilic and hydrophobic parts etc. In this context, attempts were made to improve the structural features of polyanionic copolymers. As a background, a major problem for the polyanionic diblock copolymers in Fig. 4 was assumed to arise from that charge density for a given molecular weight of the molecule is too high. Thus, the structural optimization was focused on reducing the charge density of the polyanionic copolymers.

One approach is to synthesize triblock copolymer, similar to the polyanionic diblock copolymers in Fig. 4 , but with two buoyant blocks and a central anchoring block (similarly to "Pluronic ${ }^{\circledR}$ "). This is to keep one buoyant block as charged whereas the other buoyant block as neutral, for instance, PEG- $b$-PMEA- $b$-PMAA, so that the charge density of the copolymer, as well as on the nonpolar surface can be enforced to be halved [29]. Another approach is to copolymerize polyanions chains onto a hydrophobic backbone, for example, poly(2-hydroxyethyl methacrylate) (pHEMA), and thus form graft copolymers instead of forming block copolymers. Higher bonding stability of graft copolymers compared to block copolymers is arising from multiple anchoring from the anchoring groups on the surface, and has been confirmed by many previous studies [30,31]. Additionally, the charge density of polyanions chains, PMAA, was also further reduced by random copolymerization of PMAA with PMEA. Eventually, the copolymer was in the form of PHEMA-g-(PMEA-ran-PMAA) [32]. Both approaches indeed showed improved surface adsorption and concurrently improved aqueous lubricating properties at neutral $\mathrm{pH}$, and the major driving force for this improvement is thought to be dilution of large charge density of polyelectrolyte chains on the nonpolar surface. Apparently, these two attempts adopted very limited variation of the structural features of polyaninoic copolymers, and much larger pool of parameters can be further manipulated in future studies. It must be also pointed out that the lubricating efficacy of these new polyanionic copolymers, despite the improvement compared to the simple PAA-based copolymers in Fig. 4, is not necessarily superior to their neutral counterparts [29]. Whether polyelectrolyte chains can be an effective solution to improve the load-carrying capacity of water as lubricant still remains inconclusive for freely adsorbing/detaching lubricant additives.

\section{Conclusion}

In this paper, a question was addressed whether the charged polymers can still show superior aqueous lubricating properties to neutral counterpart when they are employed as "free" surface-active additives for nonpolar sliding interface. Charges along the polymer 
chains are significant as it is one of viable means to improve poor pressure-handling problems of water as lubricant. Firstly, at nonpolar, hydrophobic PDMSPDMS sliding interface, all the polyanions employed in this study, including BSM, a representative biopolymer that is responsible for the lubrication of biological tissues, as well as the polyanionic diblock copolymers, showed improved aqueous lubrication at low $\mathrm{pH}$ and/ or with high ionic strength, i.e. when charge densities along the polyelectrolytes are rather reduced. This is mainly due to that the electrostatic repulsion between polyanions on nonpolar substrate is reduced at low $\mathrm{pH}$ and/or high salt condition. Nevertheless, a major difference between biopolymer (BSM) and synthetic copolymers (the polyanioic diblock copolymers) is that the former is still effective even at neutral $\mathrm{pH}$ where the charge moieties are fully active. On the other hand, the diblock copolymers synthesized in this study revealed extremely poor aqueous lubricating properties in neutral $\mathrm{pH}$ condition, which was ascribed to that charge density for the anionic diblock copolymers was too high. Further structural control to reduce the charge density of the synthetic polyanions successfully improved the adsorption and aqueous lubricating properties at neutral pH condition. However, relatively inferior aqueous lubricating capabilities of charged polymers to neutral counterparts remained consistent.

\section{References}

[1] Lee, S., Spencer, N. D., Achieving Ultra-low Friction by Aqueous, Brush-Assisted Lubrication. In: Erdemir, A., Martin, J.-M. (eds.) Superlubricity, Elsevier, The Netherlands, pp. 365-396, 2007. (ISBN: 978-0-44452772-1)

[2] Klein, J., "Hydration Lubrication", Friction, Vol. 1, pp. 1-23, 2013.

[3] Dai, Z., Tong, J., Ren, L., "Researches and Developments of Biomimetics in Tribology", Chines Science Bulletin, Vol. 51, pp. 2681-2689, 2006.

[4] Lee, S., Spencer, N. D., "Sweet, Hairy, Soft, and Slippery", Science, Vol. 319, 575-576.

[5] Dedinaite, A., Pettersson, T., Mohanty, B., Claesson, P. M., "Lubrication by Organized Soft Matter", Soft Matter, Vol. 6, pp. 1520-1526, 2010.

[6] Stachowiak, G., Batchelor, A. W., Engineering Tri- bology, 3rd Ed, Butterworth-Heinemann, 2005. (ISBN 978-0-7506-7836-0)

[7] Klein, J., Kumacheva, E., Mahalu, D., Perahia, D., Fetter, L.J., "Reduction of Frictional Forces between Solid Surfaces Bearing Polymer Brushes", Nature, Vol. 370, pp. 634-636, 1994.

[8] Zhao, B., Brittain, W. J., "Polymer Brushes: Surface Immobilized Macromolecules", Prog. Polym. Sci., Vol. 25, pp. 677-710, 2000.

[9] Rühe, J. Polymer Brushes: Chap. 1, On the Way to Tailor-made Surfaces. In: Advincular, R. C., Brittain, W. J., Caster, K. C., Rühe, J. (eds.) Polymer Brushes: Synthesis, Characterization, Applications, Wiley-VCH Verlag GmbH \& Co. KGaA, Weinheim, 2004. (ISBN: 9783527603824)

[10] Klein, J., "Shear, Friction, and Lubrication Forces between Polymer-Bearing Surfaces", Annu. Rev. Mater. Sci., Vol. 26, pp. 581-612, 1996.

[11] Raviv, U., Giasson, S., Kampf, N., Gohy, J.-F., Jerome, R., Klein, J., "Lubrication by Charged Polymers", Nature, Vol. 319, No. 5863, pp. 575-576, 2008. Lee, S., Spencer, N. D., "Sweet, Hairy, Soft, and Slippery", Nature, Vol. 425, pp. 163-165, 2003.

[12] Liberelle, B., Giasson, S., "Friction and Normal Interaction Forces between Irreversibly Attached Weakly Charged Polymer Brushes", Langmuir, Vol. 24, pp. 1550-1559, 2008.

[13] Kobayashi, M., Terayama, Y., Hosaka, N., Kaido, M., Suzuki, A., Yamada, N., Torikai, N., Ishihara, K., Takahara, A., "Friction Behavior of High-Density poly(2-methaarcryloxyethyl phophorylcholine) Brush in Aqueous Media”, Soft Matter, Vol. 3, pp. 740746, 2007.

[14] Kobayashi, M., Takahara, A., "Tribological Properties of Hydrophilic Polymer Brushes Under Wet Conditions", Science, Vol. 319, No. 5863, pp. 575576, 2008. Lee, S., Spencer, N. D., "Sweet, Hairy, Soft, and Slippery", The Chem. Rec., Vol. 10, pp. 208-216, 2010.

[15] Heeb, R., Bielecki, R. B., Lee, S., Spencer, N. D., "Room-Temperature, Aqueous-Phase Fabrication of Poly(metharcrylic acid) Brushes by UV-LED-Induced, Controlled Radical Polymerization with High Sensitivity for Surface-Bound Species", Macromolecules, Vol. 42, pp. 9124-9132, 2009.

[16] Røn, T., Javakhishvili, I., Jankova, K., Hvilsted, S., Lee, S., "Adsorption and Aqueous Lubricating Properties of Charged and Neutral Amphiphic Diblock Copolymers at a Compliant, Hydrophobic Interface", Langmuir, Vol. 29, pp. 7782-7792, 2013.

[17] Höök, F., Vörös, J., Rodahl, M., Kurrat, R., Böni, P., Ramsden, J. J., Textor, M., Spencer, N. D., “A Comparative Study of Protein Adsorption on Titanium 
Oxide Surfaces Using in situ Ellipsometry, Optical Waveguide Lightmode Spectroscopy, and Quartz Crystal Microbalance/Dissipation", Colloids and Surfaces B: Biointerfaces, Vol. 24, pp. 155-170, 2002.

[18] Kurrat, R., Textor, M., Ramsden, J. J., Spencer, N. D., "Instrumental Improvements in Optical Waveguide Lightmdoe Spectroscopy for the Study of Biomolecule Adsorption", Rev. Sci. Instrum., Vol. 68, pp. 2172-2176, 1997.

[19] de Feijter, J. A., Benjamins, J., Veer, F. A., "Ellipsometry as a Tool to Study Adsorption Behavior of Synthetic and Biopolymers at Air-Water-Interface", Biopolymers, Vol. 17, pp. 1759-1772, 1978.

[20] Lee, S., Müller, M., Heeb, R., Zürcher, S., Tosatti, S., Heinrich, M., Amstad, F., Pechmann, S., Spencer, N. D., "Self-Healing Behavior of a Polyeletrolytebased Lubricant Additive for Aqueous Lubrication of Oxide Materials", Tribol. Lett., Vol. 24, pp. 217223, 2006.

[21] Desseyn, J.-L., Tetaert, D., Gouyer, V., “Architecture of the Large Membrane-bound Mucins", Gene, Vol. 410, pp. 215-222, 2008.

[22] Thornton, D., Rousseau, K., McGuckin, M. A., "Structure and Function of the Polymeric Mucins in Airways Mucus", Ann. Rev. Physiol., Vol. 70, pp. 459-486, 2008.

[23] Hattrup, C. L., Gendler, S. J., "Structure and Function of the Cell Surface (Tethered) Mucins", Ann. Rev. Physiol., Vol. 70, pp. 431-457, 2008.

[24] Shi, L., Caldwell, K. D., "Mucin Adsorption to Hydrophobic Surfaces", J. Col. Interf. Science, Vol. 224, pp. 372-381, 2000.

[25] Stuart, M. A. C., Hoogendam, C. W., de Keizer, A., "Kinetics of Polyelectrolyte Adsorption", J. Phys. Condensed Matter, Vol. 9, pp. 7767-7783, 1997.
[26] Abraham, T., Giasson, S., Gohy, J. F., Jerome, R., Müller, B., Stamm, M., "Adsorption Kinetics of a Hydrophobic-Hydrophilic Diblock Polyelectrolyte at the Solid-Aqueous Solution Interface: A Slow Birth and Fast Growth Process", Macromolecules, Vol. 33, pp. 6051-6059, 2000.

[27] Nikorgeorgoes, N., Madsen, J. B., "Influence of Impurities and Contact Scale on the Lubricating Properties of Bovine Submaxillary Mucin (BSM) Films on a Hydrophobic Surface", Colloids and Surfaces B: Biointerfaces, submitted, 2014.

[28] Sotres, J., Madsen, J. B., Arnebrant, T., Lee, S., “Adsorption and Nanowear Properties of Bovien Submaxillary Mucin Films on Solid Surfaces: Influence of Solution pH and Substrate Hydrophobicity", J. Coll. Interf. Sci., Vol. 428, pp. 242-250, 2014.

[29] Røn, T., Javakhishvili, I., Patil, N. J., Zappone, B., Hvilsted, S., Lee, S., "Aqueous Lubricating Properties of Charged (ABC) and Neutral (ABA) Triblock Copolymer Chains", Polymer, Submitted, 2014.

[30] Bijsterbosch, H. D., Stuart, M. A., Fleer, G. J., "Effect of Block and Graft Copolymers on the Stability of Colloidal Silica", J. Coll. Interf. Sci. Vol. 210, pp. 37-42, 1999.

[31] Rieger, J., Passirani, C., Benoit, J. P., Van Butsele, K., Jerome, R., Jerome, C., "Synthesis of Amphiphilic Copolymers of Poly(ethylene Oxide) and Poly(e-caprolactone) with Different Architectures, and Their Role in the Preparation of Stealthy Naoparticles", $A d v$. Funct. Mater, Vol. 16, pp. 1506-1514, 2006.

[32] Javakhishvili, I., Røn, T., Jankova, K., Hvilsted, S., Lee, S., "Synthesis, Characterization, and Aqueous Lubricating Properties of Amphiphilic Graft Copolymers Comprising 2-Methoxyethyl Acrylate", Science, Vol. 47, pp. 2019-2029, 2014. 